\title{
TOWARDS NEW ANTITUBERCULAR DRUGS
}

\author{
CLAUDIA BINDA (*), LAURENT CHIARELLI (*), \\ MARIA ROSALIA PASCA $(*)$, ANDREA MATTEVI $(*)$
}

Nota presentata dal s.c. Andrea Mattevi

(Adunanza del 17 gennaio 2013)

\begin{abstract}
SunTO. - La tubercolosi, con milioni di nuovi casi ogni anno, rappresenta tuttora una preoccupante minaccia per la salute mondiale. Questa malattia infettiva è causata dal Mycobacterium tuberculosis e colpisce principalmente i polmoni, sebbene possa diffondersi anche ad altri organi. La particolare parete cellulare dei micobatteri li rende resistenti alla maggior parte degli antibiotici e per questo motivo gli unici farmaci di prima linea ad essere efficaci risalgono a parecchi decenni fa. La recente comparsa di ceppi resistenti a questi farmaci ha reso la ricerca di nuove terapie antitubercolari sempre più urgente. Nell'ambito di una rete di gruppi di ricerca finanziata dalla Comunità Europea, una nuova classe di agenti antitubercolari, i benzotiazinoni (BTZs), è stata scoperta nel 2009 portando all'identificazione di un nuovo bersaglio coinvolto nella biosintesi della parete cellulare dei micobatteri. Questo enzima è la decaprenilfosforil- $\beta$-D-ribosio 2'epimerasi (DprE1) che catalizza un passaggio essenziale nella sintesi dell'arabinogalattano, uno dei componenti della parete cellulare. I nostri gruppi di ricerca hanno svolto approfonditi studi biochimici e strutturali su la DprE1 di M. smegmatis con lo scopo di caratterizzare il meccanismo di azione dei composti BTZ e di fornire utili elementi per l'ottimizzazione degli stessi come futuri farmaci antitubercolari.
\end{abstract}

ABSTRACT. - Tuberculosis, with million new cases every year, still represents a worldwide health threat. This infectious disease is caused by Mycobacterium tuberculosis and affects primarily the lungs, although it can spread to other organs. The peculiar

(*) Dipartimento di Biologia e Biotecnologie "Lazzaro Spallanzani”, Università di Pavia, Pavia, Italy.

E-mail: andrea.mattevi@unipv.it 
mycobacterial cell wall makes these bacteria resistant to most of the antibiotics and for this reason the first-line effective drugs now available date back to several decades ago. The recent appearance of drug-resistant strains makes the search for new antitubercular therapies more and more urgent. In the framework of a research network funded by the European Commission, a novel class of antitubercular agents, the benzothiazinones (BTZs), were discovered in 2009, which led to the identification of a new enzyme target that is involved in the biosynthesis of the mycobacterial cell wall. This enzyme is the decaprenylphosphoryl- $\beta$-D-ribose 2'epimerase (DprE1) which catalyzes an essential step in the biosynthesis of arabinogalactan, one of the mycobacterial cell components. We carried out a thorough biochemical and structural characterization of DprE1 from M. smegmatis with the aim to investigate BTZ mode of action and provide hints for drug optimization.

\section{INTRODUCTION}

Tuberculosis (TB) is an infectious disease caused by Mycobacterium tuberculosis, which is an aerobic pathogenic bacterium that establishes its infection usually in the lungs (pulmonary TB), but can also affect other sites (extrapulmonary TB). It is almost exclusively transmitted from a patient with infectious pulmonary tuberculosis via aerosolized droplets by coughing, sneezing or even speaking. Once inhaled, the bacilli are phagocytized in the air spaces primarily by resident alveolar macrophages and dendritic cells. At this point, the infection can be either eliminated by the host immune response by innate immunity, or retained as latent tuberculosis infection by adaptive immunity, or it can progress to active clinical disease by the break-down of the immune defence [1]. M. tuberculosis-infected phagocytes can migrate from the alveolar space into the lung interstitium and then, via the lymphatic and hematogenous routes, eventually disseminate to other organs [2,3]. Subsequently, the bacilli may grow unimpeded within host macrophages, resulting in primary progressive disease or reactivation disease after a short period of latency, as seen in about $10 \%$ of immune competent individuals. Alternatively, bacillary growth may be controlled, and the bacteria may be killed or may adapt to survival within cellular granulomas in a non-replicating state, thereby establishing a latent infection, as seen in approximately $90 \%$ of healthy hosts. Latent infection can persist for decades after exposure to $M$. tuberculosis before reactivating to cause active disease (primarily in the lungs), when the immune-mediated control of bacillary growth fails, as seen, for example, in patients 
with immunodeficiency virus (HIV) infection [4]. Currently, HIV infection represents the major risk for the progression of a TB latent infection into the active disease but, in the recent years, the resurgence of TB can be ascribed also to other factors such as large scale migration, transmission in overcrowded public places, and the spreading of M. tuberculosis drug resistant strains. The incidence of tuberculosis worldwide in 2012 was 8.6 million of people, with 1.3 million deaths including 320.000 people co-infected with HIV [5]. World Health Organization (WHO) estimates that about 2 billion people have latent tuberculosis and more than 100 million of these people will develop active tuberculosis [6].

The intrinsic resistance of $M$. tuberculosis to antibiotics is primarily attributed to its peculiar cell wall. This envelope contains three covalently linked components: peptidoglycan, arabinogalactan, and mycolic acid. The arabinogalactan (AG) consists of covalently coupled galactan and arabinan polymers [8]. The branched arabinan chains containing $\alpha$-D-arabinofuranosyl (Araf) are attached through the $\beta$-D-galactofuranose units. The $\alpha$-mycolates are the most abundant mycolic acids found in M. tuberculosis. Other important components of the mycobacterial cell wall are the free glycolipids, such as: trehalose dimycolate, named also cord factor, trehalose monomycolate, phthiocerol dimycocerosate and lipoarabinomannan [7].

The most important and the oldest antitubercular drug, isoniazid, targets the biosynthesis of mycolic acids, fundamental component of the cell wall. This first-line drug is the most used in TB therapy.

In fact, the treatment for drug-sensitive tuberculosis lasts at least 6 months and four first-line oral drugs (rifampicin, isoniazid, pyrazinamide, and ethambutol) must be swallowed together during the first 2 months of therapy, and two (rifampicin and isoniazid) for the subsequent 4 months. These therapies are associated with increased mortality and can induce drug-resistance [9]. In 2012, the estimated number of cases of multidrug resistant tuberculosis (MDRTB) was 450.000, with about 170.000 deaths [10]. The M. tuberculosis MDR strains are resistant to rifampicin and isoniazid, the two firstline drugs. Patients affected with MDR-TB use a combination of second-line and third-line antitubercular drugs, which are much more toxic, expensive, and less active than the first-line ones.

Unfortunately, M. tuberculosis extensively drug-resistant (XDR) isolates are resistant to rifampicin, isoniazid, fluoroquinolone, and at 
least to one of the three injectable second-line drugs: amikacin, kanamycin, or capreomycin. These XDR strains have been already reported in 92 countries [10] worldwide. Moreover, totally drug-resistant tuberculosis (TDR-TB) was described, although the exact definition of TDR-TB remains to be defined by WHO [11]. TDR-TB involves $M$. tuberculosis strains that are resistant in vitro to all firstand second-line drugs tested (isoniazid, rifampicin, streptomycin, ethambutol, pyrazinamide, ethionamide, PAS, cycloserine, ofloxacin, amikacin, ciprofloxacin, capreomycin and kanamycin) [11]. Two patients with TDR-TB were first identified in Italy in 2003 [12] and, more recently, four cases of TDR-TB were discovered in India [13]. Three new antitubercular drugs, bedaquiline, delamanid and linezolid have been approved by the US Food and Drug Administration (FDA) and the European Medicines Agency to be used as therapy for TDR-TB [11].

New antitubercular drugs, as well as novel cellular targets, are urgently required to overcome the problem of drug resistance and to tackle the worldwide spreading of tuberculosis. In the last few years, there have been several new antitubercular drugs in preclinical and clinical trials, after a long period without novelties [14] (Tab. 1). Among the drugs in clinical development bedaquiline was approved by the FDA (Food and Drug Administration) in December 2012 as part of the combination therapy for the treatment of adult patients affected by drug-resistant tuberculosis. Bedaquiline is very active also against latent tuberculosis and its cellular target is the ATP synthase [15]. The mycobacterial ATP synthase is 20.000 fold more sensitive to this drug than the human mitochondrial ATP synthase, confirming that it is a good drug target [16]. A very promising new compound, PBTZ169 (piperazine-containing benzothiazinones; PBTZ) is currently in the last phase of preclinical trials (Fig. 1) [17].

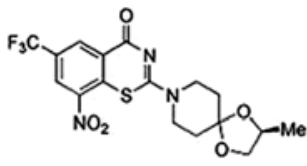

BTZ043

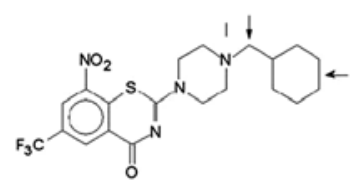

PBTZ169

Fig.1 - BTZ043 and PBTZ169 antitubercular compounds.

The arrows indicate the sites for further drug optimization. 
This molecule is a new derivative of the benzothiazinones (BTZs) class of compounds which were discovered in 2009 (see section 2) to inhibit a novel target, the $M$. tuberculosis decaprenylphosphoryl- $\beta$-D-ribose 2'oxidase (DprE1) [18,19]. This represents an essential enzyme involved in the synthesis of the arabinogalactan moiety of the mycobacterial cell wall. Herewith, we review the work that was performed by our and other groups on the molecular and structural characterization of DprE1 and the BTZ inhibitors as novel candidates in the development of new treatments for tuberculosis.

Tab. 1 - New drugs in preclinical and clinical development

(modified from http://www.newtbdrugs.org/pipeline.php) [11].

\begin{tabular}{llll}
\hline $\begin{array}{l}\text { Preclinical/ } \\
\text { Clinical trials }\end{array}$ & Drug & Drug class & Mode of action/target \\
\hline Last stages of & BTZ043 & Benzothiazinones & DprE1 \\
preclinical trials & CPZEN-45 & Caprazene nucleoside & WecA (cell wall) \\
& DC-159a & Fluoroquinolones & Block of DNA synthesis \\
& PBTZ169 & Benzothiazinones & DprE1 \\
& Q203 & Imidazopyridine & Cytochrome bc1 \\
& SQ609 & Dipiperidine & Cell wall biosynthesis \\
& SQ641 & Capuramycin & TL1 (cell wall) \\
& TBI-166 & Riminophenazines & Not known \\
& TBA-354 & Nitroimidazole & Generate NO \\
\hline Clinical Phase II & AZD5847 & Oxazolidinones & 23S rRNA \\
& BEDAQUILINE & Diarylquinolines & ATP synthase \\
& DELAMANID & Nitroimidazole & Generate NO \\
& LINEZOLID & Oxazolidinones & 23S rRNA \\
& PA-824 & Nitroimidazole & Generate NO \\
& RIFAPENTINE & Rifamycin & RNA polymerase \\
& SQ-109 & 1,2-diamine & MmpL3 \\
SUTEZOLID & Oxazolidinones & 23S rRNA \\
\hline BEDAQUILINE & Diarylquinolines & ATP synthase \\
GATIFLOXACIN & Fluoroquinolones & Block of DNA synthesis \\
& MOXIFLOXACIN & Fluoroquinolones & Block of DNA synthesis \\
RIFAPENTINE & Rifamycin & RNA polymerase \\
\hline Clinical Phase III & &
\end{tabular}

${ }^{1}$ For drug-sensitive-TB; ${ }^{2}$ for MDR-TB; ${ }^{3}$ for latent TB. 


\section{The DisCOVERY OF BENZOTHIAZINONES AND DpRE1}

In the last years two projects which involve our groups at the University of Pavia have been funded by the European Commission: "New Medicines for Tuberculosis" (NM4TB; VI Framework program) and "More Medicines for tuberculosis" (MM4TB; VII Framework program). Within these projects, the BTZ novel class of antitubercular was discovered and their molecular target, the M. tuberculosis DprE1 enzyme, was identified and characterized. BTZs showed a nanomolar bactericidal activity against mycobacteria growing in in vitro and in ex vivo models [18]. In murine models of acute or chronic TB, the preclinical candidate BTZ043 showed efficacy comparable with that observed with INH and RIF [18]. BTZ043 is active against M. tuberculosis drugsusceptible, MDR and XDR clinical isolates [20].

The M. tuberculosis dprE1 (or Rv3790) gene encodes the essential enzyme DprE1, involved in the biosynthesis of arabinogalactan, an essential component of the mycobacterial cell wall core [19]. DprE1 works in concert with DprE2 (encoded by the gene dprE2) and both enzymes compose the decaprenylphosphoryl- $\beta$-D-ribose 2 'epimerase, which is involved in the biosynthesis of the arabinan moiety of arabinogalactan [21]. In particular, DprE1 catalyzes the first step in the epimerization reaction of decaprenylphosphoryl- $\beta$-D-ribose (DPR) into decaprenol-phosphoryl- $\beta$-D-arabinose (DPA), which represents the only donor of arabinofuranose residues for arabinogalactan biosynthesis in mycobacteria [21]. Therefore, it has been suggested that DprE1 acts as decaprenylphosphoryl- $\beta$-D-ribose oxidase and DprE2 as a decaprenylphosphoryl- $\beta$-D-2-keto erythro pentose reductase. Both the dprE1 and dprE2 genes are essential in M. tuberculosis H37Rv [22].

The discovery of BTZs and DprE1 was accomplished by the phenotypic screening approach which represents the strategy adopted by NM4TB and MM4TB to unveil new antitubercular therapies. This involves testing of libraries of compounds in mycobacterial cell cultures followed by isolation of resistant mutants to identify the molecular target of those molecules with bactericidal activity. In particular, spontaneous mutants bearing the substitution of the Cys387 (to Ser or Gly) residue of $M$. tuberculosis DprE1 were found to be responsible for BTZ resistance. This cysteine residue is highly conserved in DprE1 orthologous proteins from different BTZ- susceptible Actinobacteria. Only in a few species such as Mycobacterium avium and Mycobacterium aurum, 
Cys 387 residue is replaced by Serine or Alanine, respectively, which render these species naturally resistant to BTZs [18]. New DprE1 inhibitors were recently discovered in these last few years [23-29], underlying the high vulnerability of this M. tuberculosis cellular target.

\section{The DPrE1 CRYSTAL STRUCTURE AND THE MECHANISM OF BENZOTHIAZINONES INHIBITION}

Despite the potent anti-tubercular activity of BTZ043 in mycobacterial cell cultures and in ex vivo models of the disease, its in vivo efficacy in mice is much lower. The observation that analogues of BTZ043 in which the nitro group is replaced by an amino group are much less active even in vitro (500- to 5000-fold less potent than that of BTZ043) [18] demonstrated that the nitro group is essential to inhibit the DprE1 target. This observation suggested that in mice endogenous nitroreductases might reduce the nitro group to an amino group, providing an explanation for the lower potency of this compound in mice. Indeed, it was demonstrated that the overexpression of the flavin-dependent nitroreductase NfnB in M. smegmatis represents a mechanism of BTZ resistance due to drug inactivation by reduction of the nitro group [30]. To further investigate the BTZs mode of action and to provide drug design guidelines in view of the use of this class of compounds as effective antitubercular drugs, it was essential to undertake the molecular characterization of BTZ043 inhibition and of its target enzyme DprE1.

The initial discovery of BTZ043 and DprE1 through the phenotypic screening, which highlighted the Cys387 mutation in DprE1 as a mechanism of resistance (see section 2), led to the hypothesis that the compound might represent a prodrug which requires some sort of activation to inhibit DprE1 by involving a covalent interaction with the enzyme itself. This idea was supported by mutagenesis and mass spectrometry analysis experiments performed in the framework of the MM4TB network, which proposed the formation of a semimercaptal adduct between BTZ043 and Cys387 as a possible mechanism of DprE1 inactivation [31].

We carried out a thorough biochemical and crystallographic investigation of DprE1 to determine its enzymatic properties and to obtain a model of the three-dimensional structure at atomic level [32]. To accomplish this task we produced the protein DprE1 from M. smegmatis (83\% identical to the M. tuberculosis homolog) in its recombinant form by 
cloning the corresponding cDNA in a vector for the expression in E. coli bacterial cultures. The protein was then extracted from the cells and purified by chromatographic techniques, yielding a bright yellow pure sample due to the presence of the FAD cofactor bound in its oxidized state. We developed two assays to measure the DprE1 enzymatic activity by using farnesylphosphoryl- $\beta$-D-ribofuranose (FPR) as substrate, which is a watersoluble analogue of the DPR natural substrate of the enzyme with a shorter aliphatic chain. The first assay is based on a widely used peroxidase-coupled spectrophotometric method which measures the production of hydrogen peroxide. This represents the secondary product of the enzymatic reaction resulting from the reoxidation of the FAD cofactor by molecular oxygen, a typical property of the oxidase class of enzymes. We determined a $\mathrm{k}_{\mathrm{cat}}$ of $3.1 \mathrm{~min}^{-1}$ (i.e. the number of catalytic cycles per minute), which appeared to be low compared to other oxidases. Therefore, we probed other organic molecules which might act as alternative electron acceptors. We found that menaquinone can reoxidize the DprE1 cofactor and, being a natural component of the bacterial membranes, it may represent the physiological electron acceptor of this enzyme. Moreover, among artificial organic molecules, we found that 2,6-dichlorophenolindophenol (DCPIP) can reoxidize the DprE1 FAD cofactor more efficiently than molecular oxygen, with a 4-fold higher $\mathrm{k}_{\text {cat }}$ value. This reaction can be monitored spectrophotometrically, providing a second method to measure the DprE1 enzymatic activity. By using these assays we could perform a comprehensive biochemical characterization of the DprE1 target, including the determination of the inhibition parameters of BTZ043 and its analogues. Although these parameters cannot be directly compared to the minimal inhibitory concentration (MIC) values determined on mycobacterial cultures because they refer to completely different experimental conditions, these assays provide a convenient method to test libraries of compounds for their capacity to bind and inhibit DprE1 with the aim to find other potential candidates for anti-tuberculosis therapies.

In parallel to these biochemical studies, we carried out an extensive screening to obtain high quality crystals of DprE1 to be used in Xray diffraction experiments for the elucidation of the three-dimensional structure of the target enzyme. We could grow cube-shaped yellow crystals which allowed us to solve the DprE1 structure in its FADbound native form at $2.1 \AA$ resolution (Fig. 2). The DprE1 polypeptide chain folds into a two-domain topology globular structure: one domain binds the FAD cofactor in a non-covalent form (in yellow in Fig. 2), 
whereas the other domain contains a cavity (light blue in Fig. 2) that lies at the interface with the isoalloxazine ring of the FAD cofactor. This cavity is shielded from the outside by a loop (residues 320-329) and represents the active site of the enzyme. The Cys394 residue, corresponding to Cys387 in M. tuberculosis which proved to be essential for BTZ antitubercular activity, is located on the rim of the cavity. Using crystals obtained in another experimental condition in the presence of BTZ043, we determined the DprE1 crystal structure in complex with the inhibitor (inset in Fig. 2). This could be unambiguously modeled in the active site forming a covalent bond with the Cys394 side chain, which ultimately proved the formation of the semimercaptal adduct observed in mass spectrometry experiments. BTZ043 binds with its benzothiazinone moiety located in front of the flavin and the spyrocyclic ring extending outside of the protein surface, resulting in a disordered conformation of the loop 320-329 (inset in Fig. 2).

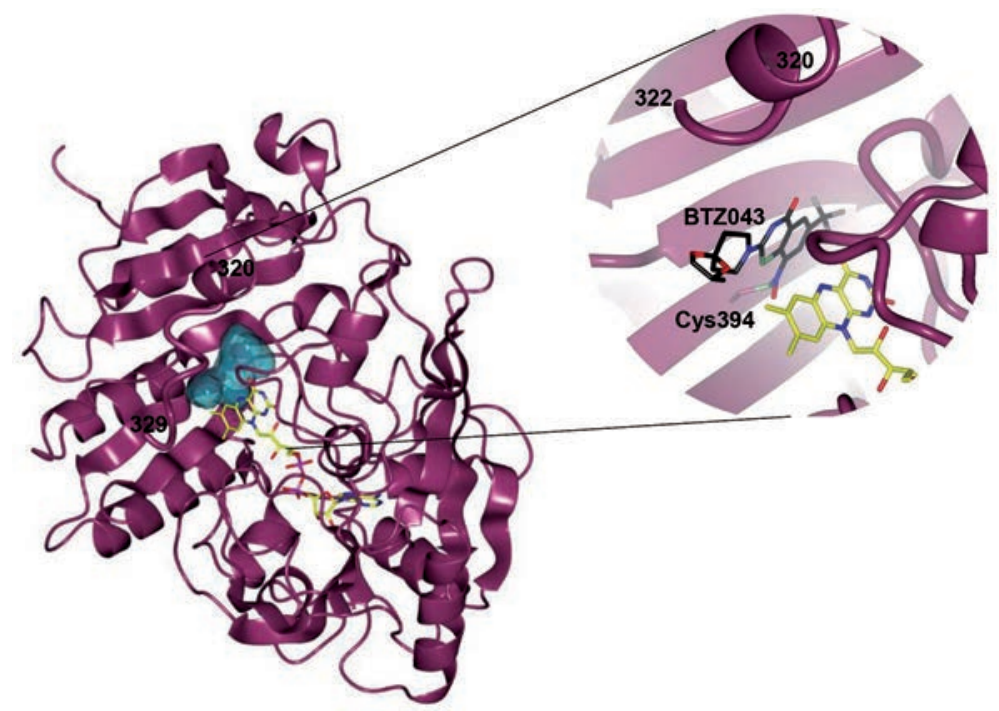

Fig. 2 - Crystal structure of M. smegmatis DprE1, the molecular target of the antitubercular BTZs. The structure is represented as dark purple ribbon, with the bound FAD cofactor (carbon, nitrogen, oxygen and phosphour atoms are yellow, blue, red and magenta, respectively). The active site cavity is drawn as semitransparent light blue surface. The active site loop 320-329 is highlighted as bold ribbon. Inset: zoomed view of the DprE1 active site with the BTZ043 bound (carbon, nitrogen, oxygen, sulfur, fluorine atoms are black, blue, red, green, gray, respectively). The covalent bond between Cys394 and BTZ043 is shown. 
Interestingly, the BTZ043 adduct was observed only when crystals were grown also in the presence of the FPR substrate. This demonstrated that the reduced FAD cofactor (resulting from the catalytic reaction with FPR) converts the nitro group of the inhibitor to a nitroso derivative that is able to react with the Cys394 thiol group leading to the observed covalent adduct. This mechanism was further demonstrated by the observation that, similarly to menaquinone, BTZ043 is able to reoxidize the reduced FAD cofactor of DprE1 in anaerobic conditions. Therefore, BTZ043 represents a suicide prodrug which requires an enzyme-dependent activation to irreversibly inhibit DprE1 (Fig. 3).

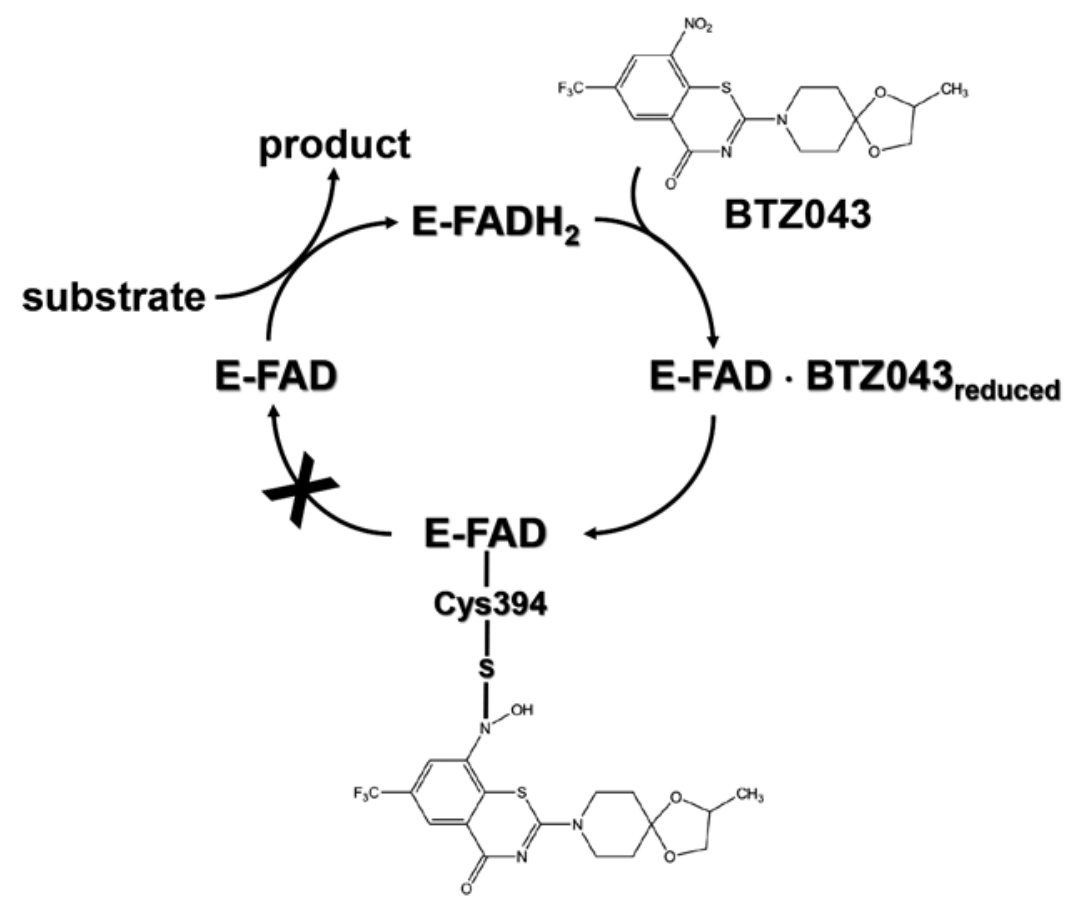

Fig. 3 - Scheme describing the suicide mode of action of BTZ043 targeting DprE1.

This highlights the strategy adopted within the MM4TB network for the identification of new antitubercular agents by screening compounds in M. tuberculosis cell cultures rather than in high-throughput biochemical assays extrapolated from the cell environment. Despite BTZs feature negative properties such as inactivation by nitroreduc- 
tases, they provided a convenient scaffold for drug optimization. As described in the previous sections, the piperazine derivatives of BTZs (such as PBTZ169) showed improved features such as lower toxicity, better pharmacokinetics and lower susceptibility to nitroreductases as gathered from the higher antitubercular activity in murine models of the disease [17]. More studies are ongoing to further optimize BTZs and identify new molecules targeting DprE1.

\section{ACKNOWLEDGEMENTS}

This work was supported by the European Community within the FP7/2007-2013 Programme.

\section{REFERENCES}

[1] M. Gengenbacher, S.H. Kaufmann, Mycobacterium tuberculosis: success through dormancy, FEMS Microbiol. Rev. 36 (2012), 514-532.

[2] T.R. Frieden, T.R. Sterling, S.S. Munsiff, C.J. Watt, C. Dye, Tuberculosis, Lancet 362 (2003), 887-899.

[3] S.H. Kaufmann, New issues in tuberculosis, Ann. Rheum. Dis. 63(2004), ii50-ii56.

[4] E. Nuermberger, W.R. Bishai, J.H. Grosset, Latent tuberculosis infection, Semin. Respir. Crit. Care Med. 25 (2004), 317-336.

[5] WHO. Treatment of tuberculosis guidelines. $4^{\text {th }}$ edition. Geneva: World Health Organization, 2010. http://whqlibdoc.who.int/publications/2010/9789241547833 _eng.pdf.

[6] N.D. Walter, J. Painter, M. Parker, P. Lowenthal, J. Flood, Y. Fu, R. Asis, R. Reves; Tuberculosis Epidemiologic Studies Consortium, Persistent latent tuberculosis reactivation risk in us immigrants. Am. J. Respir. Crit. Care Med. 189 (2014), 88-95.

[7] D. Kaur, M.E. Guerin, H. Skovierová, P.J. Brennan, M. Jackson. Biogenesis of the cell wall and other glycoconjugates of Mycobacterium tuberculosis. Adv. Appl. Microbiol. 69 (2009), 23-78.

[8] L.G. Dover, L.J. Alderwick, A.K. Brown, K. Futterer, G.S. Besra, Regulation of cell wall synthesis and growth, Curr. Mol. Med. 7(2007), 247-276.

[9] A.I. Zumla, S.H. Gillespie, M. Hoelscher, P.P. Philips, S.T. Cole, I. Abubakar, T.D. McHugh, M. Schito, M. Maeurer, A.J. Nunn, New antituberculosis drugs, regimens, and adjunct therapies: needs, advances, and future prospects, Lancet Infect. Dis. 14 (2014), 327-340.

[10] WHO. Global Tuberculosis report 2013. WHO/HTM/TB/2013. http://www. who.int/tb/publications/global_report/en (accessed Nov 12, 2013). 
[11] S.K. Parida, R. Axelsson-Robertson, M.V. Rao, N. Singh, I. Master, A. Lutckii, S. Keshavjee, J. Andersson, A. Zumla, M. Maeurer, Totally drug-resistant tuberculosis and adjunct therapies, J. Intern. Med. (2014). doi: 10.1111/joim.12264.

[12] G.B. Migliori, G. De Iaco, G. Besozzi, R. Centis, D.M. Cirillo. First tuberculosis cases in Italy resistant to all tested drugs. Euro Surveill. 12 (2007), E070517.1.

[13] Z.F. Udwadia, R.A. Amale, K.K. Ajbani, C. Rodrigues, Totally drug-resistant tuberculosis in India. Clin. Infect. Dis. 54 (2012), 579-581.

[14] G. Riccardi, M.R. Pasca, Trends in discovery of new drugs for tuberculosis therapy, J. Antibiot. (Tokyo), 67 (2014), 655-659.

[15] K. Andries, P. Verhasselt, J. Guillemont, H.W. Göhlmann, J.M. Neefs, H. Winkler, J. Van Gestel, P. Timmerman, M. Zhu, E. Lee, P. Williams, D. de Chaffoy, E. Huitric, S. Hoffner, E. Cambau, C. Truffot-Pernot, N. Lounis, V. Jarlier, A diarylquinoline drug active on the ATP synthase of Mycobacterium tuberculosis, Science, 307 (2005), 223-227.

[16] A.C. Haagsma, R. Abdillahi-Ibrahim, M.J. Wagner, K. Krab, K. Vergauwen, J. Guillemont, K. Andries, H. Lill, A. Koul, D. Bald. Selectivity of TMC207 towards mycobacterial ATP synthase compared with that towards the eukaryotic homologue, Antimicrob. Agents Chemother. 53 (2009), 1290-1292.

[17] V. Makarov, B. Lechartier, M. Zhang, J. Neres, A.M. van der Sar, S.A. Raadsen, R.C. Hartkoorn, O.B. Ryabova, A. Vocat, L.A. Decosterd, N. Widmer, T. Buclin, W. Bitter, K. Andries, F. Pojer, P.J. Dyson, S.T. Cole, Towards a new combination therapy for tuberculosis with next generation benzothiazinones, EMBO Mol. Med. 6 (2014), 372-383.

[18] V. Makarov, G. Manina, K. Mikusova, U. Möllmann, O. Ryabova, B. SaintJoanis, N. Dhar, M.R. Pasca, S. Buroni, A.P. Lucarelli, A. Milano, E. De Rossi, M. Belanova, A. Bobovska, P. Dianiskova, J. Kordulakova, C. Sala, E. Fullam, P. Schneider, J.D. McKinney, P. Brodin, T. Christophe, S. Waddell, P. Butcher, J. Albrethsen, I. Rosenkrands, R. Brosch, V. Nandi, S. Bharath, S. Gaonkar, R.K. Shandil, V. Balasubramanian, T. Balganesh, S. Tyagi, J. Grosset, G. Riccardi, S.T. Cole, Benzothiazinones kill Mycobacterium tuberculosis by blocking arabinan synthesis, Science, 324 (2009), 801-804.

[19] G. Riccardi, M.R. Pasca, L.R. Chiarelli, G. Manina, A. Mattevi, C. Binda, The DprE1 enzyme, one of the most vulnerable targets of Mycobacterium tuberculosis, Appl. Microbiol. Biotechnol, 97 (2013), 8841-8848.

[20] M.R. Pasca, G. Degiacomi, A.L. Ribeiro, F. Zara, P. De Mori, B. Heym, M. Mirrione, R. Brerra, L. Pagani, L. Pucillo, P. Troupioti, V. Makarov, S.T. Cole, G. Riccardi, Clinical isolates of Mycobacterium tuberculosis in four European hospitals are uniformly susceptible to benzothiazinones, Antimicrob. Agents Chemother. 54(2010), 1616-1618.

[21] B.A. Wolucka, Biosynthesis of D-arabinose in mycobacteria - a novel bacterial pathway with implications for antimycobacterial therapy, FEBS J. 275 (2008), 2691-271.

[22] C.M. Sassetti, D.H. Boyd, E.J. Rubin, Genes required for mycobacterial growth defined by high density mutagenesis, Mol. Microbiol. 48(2003), 77-84. 
[23] M. Naik, V. Humnabadkar, S.J. Tantry, M. Panda, A. Narayan, S. Guptha, V. Panduga, P. Manjrekar, L.K. Jena, K. Koushik, G. Shanbhag, S. Jatheendranath, M.R. Manjunatha, G. Gorai, C. Bathula, S. Rudrapatna, V. Achar, S. Sharma, A. Ambady, N. Hegde, J. Mahadevaswamy, P. Kaur, V.K. Sambandamurthy, D. Awasthy, C. Narayan, S. Ravishankar, P. Madhavapeddi, J. Reddy, K. Prabhakar, R. Saralaya, M. Chatterji, J. Whiteaker, B. McLaughlin, L.R. Chiarelli, G. Riccardi, M.R. Pasca, C. Binda, J. Neres, N. Dhar, F. Signorino-Gelo, J.D. McKinney, V. Ramachandran, R. Shandil, R. Tommasi, P.S. Iyer, S. Narayanan, V. Hosagrahara, S. Kavanagh, N. Dinesh, S.R. Ghorpad, 4-aminoquinolone piperidine amides: noncovalent inhibitors of DprE1 with long residence time and potent antimycobacterial activity, J Med Chem. 57 (2014), 5419-34.

[24] M. Panda, S. Ramachandran, V. Ramachandran, P.S. Shirude, V. Humnabadkar, K. Nagalapur, S. Sharma, P. Kaur, S. Guptha, A. Narayan, J. Mahadevaswamy, A. Ambady, N. Hegde, S.S. Rudrapatna, V.P. Hosagrahara, V.K. Sambandamurthy, A. Raichurkar, Discovery of pyrazolopyridones as a novel class of noncovalent DprE1 inhibitor with potent anti-mycobacterial activity, J. Med. Chem. 57(2014), 4761-4771.

[25] P.S. Shirude, R. Shandil, C. Sadler, M. Naik, V. Hosagrahara, S. Hameed, V. Shinde, C. Bathula, V. Humnabadkar, N. Kumar, J. Reddy, V. Panduga, S. Sharma, A. Ambady, N. Hegde, J. Whiteaker, R.E. McLaughlin, H. Gardner, P. Madhavapeddi, V. Ramachandran, P. Kaur, A. Narayan, S. Guptha, D. Awasthy, C. Narayan, J. Mahadevaswamy, K.G. Vishwas, V. Ahuja, A. Srivastava, K.R. Prabhakar, S. Bharath, R. Kale, M. Ramaiah, N.R. Choudhury, V.K. Sambandamurthy, S. Solapure, P.S. Iyer, S. Narayanan, M. Chatterji, Azaindoles: noncovalent DprE1 inhibitors from scaffold morphing efforts, kill Mycobacterium tuberculosis and are efficacious in vivo, J. Med. Chem. 56 (2013), 9701-9708.

[26] F. Wang, D. Sambandan, R. Halder, J. Wang, S.M. Batt, B. Weinrick, I. Ahmad, P. Yang, Y. Zhang, J. Kim, M. Hassani, S. Huszar, C. Trefzer, Z. Ma, T. Kaneko, K.E. Mdluli, S. Franzblau, A.K. Chatterjee, K. Johnsson, K. Mikusova, G.S. Besra, K. Fütterer, S.H. Robbins, S.W. Barnes, J.R. Walker, W.R. Jacobs Jr, P.G. Schultz, Identification of a small molecule with activity against drug-resistant and persistent tuberculosis, Proc. Natl. Acad. Sci. U. S. A. 110 (2013), E25102517.

[27] S.A. Stanley, S.S. Grant, T. Kawate, N. Iwase, M. Shimizu, C. Wivagg, M. Silvis, E. Kazyanskaya, J. Aquadro, A. Golas, M. Fitzgerald, H. Dai, L. Zhang, D.T. Hung, Identification of novel inhibitors of $M$. tuberculosis growth using whole cell based high-throughput screening, ACS Chem. Biol. 7 (2012), 1377-13784.

[28] S. Magnet, R.C. Hartkoorn, R. Székely, J. Pató, J.A. Triccas, P. Schneider, C. Szántai-Kis, L. Orfi, M. Chambon, D. Banfi, M. Bueno, G. Turcatti, G. Kéri, S.T. Cole, Leads for antitubercular compounds from kinase inhibitor library screens, Tuberculosis (Edinb). 90 (2010), 354-360.

[29] T. Christophe, M. Jackson, H.K. Jeon, D. Fenistein, M. Contreras-Dominguez, J. Kim, A. Genovesio, J.P. Carralot, F. Ewann, E.H. Kim, S.Y. Lee, S. Kang, M.J. Seo, E.J. Park, H. Skovierová, H. Pham, G. Riccardi, J.Y. Nam, L. Marsollier, M. 
Kempf, M.L. Joly-Guillou, T. Oh, W.K. Shin, Z. No, U. Nehrbass, R. Brosch, S.T. Cole, P. Brodin, High content screening identifies decaprenyl-phosphoribose 2 ' epimerase as a target for intracellular antimycobacterial inhibitors, PLoS Pathog. 5 (2009), e1000645.

[30] G. Manina, M. Bellinzoni, M.R. Pasca, J. Neres, A. Milano, A.L. Ribeiro, S. Buroni, H. Skovierová, P. Dianišková, K. Mikušová, J. Marák, V. Makarov, D. Giganti, A. Haouz, A.P. Lucarelli, G. Degiacomi, A. Piazza, L.R. Chiarelli, E. De Rossi, E. Salina, S.T. Cole, P.M. Alzari, G. Riccardi, Biological and structural characterization of the Mycobacterium smegmatis nitroreductase $\mathrm{NfnB}$, and its role in benzothiazinone resistance, Mol Microbiol. 77 (2010), 1172-1185.

[31] C. Trefzer, H. Škovierová, S. Buroni, A. Bobovská, S. Nenci, E. Molteni, F. Pojer, M.R. Pasca, V. Makarov, S.T. Cole, G. Riccardi, K. Mikušová, K. Johnsson, Benzothiazinones are suicide inhibitors of mycobacterial decaprenylphosphoryl$\beta$-D-ribofuranose 2'-oxidase DprE1, J. Am. Chem. Soc. 18 (2012), 912-915.

[32] J. Neres, F. Pojer, E. Molteni, L.R. Chiarelli, N. Dhar, S. Boy-Röttger, S. Buroni, E. Fullam, G. Degiacomi, A.P. Lucarelli, R.J. Read, G. Zanoni, D.E. Edmondson, E. De Rossi, M.R. Pasca, J.D. McKinney, P.J. Dyson, G. Riccardi, A. Mattevi, S.T. Cole, C. Binda, Structural basis for benzothiazinone-mediated killing of Mycobacterium tuberculosis, Sci. Transl. Med. 4 (2012), 150ra121. 